\title{
The impact of system nonlinearities in the problem of optimal PMU placement for power system state estimation
}

\author{
Mikhail Khokhlov ${ }^{1, *}$, Olga Pozdnyakova ${ }^{1}$ \\ ${ }^{1}$ Federal Research Centre "Komi Science Centre of the Ural Branch of the Russian Academy of Sciences", Institute for Socio-Economic \\ and Energy Problems of the North, 167982 Kommunisticheskaya str., 26, Syktyvkar, Russia
}

\begin{abstract}
In PMU-based state estimation, a linear measurement model with phasors of both state variables and measurements expressed in rectangular coordinates has proven efficiency. The rectangular coordinate formulation is also used in optimal PMU placement problem aimed at providing the power system state estimation with the most informative measurements. In this case, it is assumed that the linearity of the measurement model ensures the optimality of the found placement of PMUs for any steady-state operating condition of the power system. The results presented in this paper show that this is not the case.
\end{abstract}

\section{Introduction}

A wide area measurement system (WAMS) based on synchronized phasor measurement units (PMUs) enables to obtain real-time measurements of not only the magnitudes but also the phase angles of current and voltage phasors over the whole power system using a common time source for synchronization [1]. A direct measurement of phase angles has opened new opportunities to develop and improve power system state estimation algorithms used in the stability reserve monitoring system and the centralized emergency control system. First of all, it is the possibility to solve the state estimation problem in linear formulation when expressing phasors of branch currents and bus voltages in rectangular coordinates, which considerably simplifies the computation [2], improves the reliability and performance of numerical algorithms [3] and allows us to go to the formulation and solution of more complex problems, such as the generalized state estimation, when not only the states, but also the network topology, are estimated [4].

The deployment of WAMS and the transition to power system control based solely on PMU phasor measurements is a matter of time. According to web resource [5], the number of PMUs installed in Russia is approaching a thousand units, which is not yet sufficient to carry out state estimation of the Russian power system exclusively based on PMU measurements, although this is possible for small local systems [2]. In this regard, at the present time in Russia, as well as in other countries of the world, optimal PMU placement problem is being developed to provide the PMU installations to meet full observability of the power system and higher accuracy of the state estimation and at minimum cost.
The theory and methods of optimal experimental designs [6] are the basis for making decisions on the locations of PMUs to be installed in the power system with the objective of ensuring maximum accuracy of the state estimation. As with the power system state estimation, solving the optimal PMU placement problem relies on a linear measurement model with using rectangular coordinates for phasor measurements and state variables [7-10]. The advantage of a linear system is that the solution found does not depend on the power system steady-state, and once calculated it is optimal for any operating conditions. This favourably distinguishes the problem of choosing the PMU phasor measurements from the problem that deals with conventional measurements $(U, P, Q, I)$ which are nonlinearly related to the power system states [11-13]. In the latter case, it is necessary to resolve the issue of ensuring the optimality of the selected measurement set for various power system operation states.

In this paper, it is shown that the influence of system nonlinearity on the optimal solution of PMU placement problem remains irrespective of whether polar or rectangular coordinates formulation is used. The results of presented computational experiments illustrate the dependence of the solution on the power system load level and the loss of accuracy of state estimation results in the states other than that used when solving the optimal PMU placement problem.

\section{Representing phasor quantities in optimal PMU placement problem}

Let us consider a linear measurement model:

$$
z=H x+\xi
$$

\footnotetext{
* Corresponding author: hohlov@energy.komisc.ru
} 
where $H$ is a $m \times n$ matrix that relates vector $z$ of measured parameters to the vector $x$ of system state variables, and $\xi \sim N(0, R)$ is a measurement error vector having a known covariance matrix $R=\operatorname{diag}\left(\sigma_{i}^{2}\right)$.

Let us formulate the problem of choosing a set of $k$ measurements from a set of $m$ possible or potential measurements that minimizes the error in estimating some parameters:

$$
\begin{gathered}
\min _{y} \Phi(D(y)) \\
\text { s.t. } \sum_{i=1}^{m} y_{i} \leq k \\
y_{i} \in\{0,1\}, \quad i=1, \ldots, m
\end{gathered}
$$

where $y_{i}=1$, if the $i$ th measurement is selected, and $y_{i}=0$ otherwise, $\Phi(D(y))$ is a convex function [10] of the covariance matrix of the estimates of state variables

$$
D(\hat{x}, y)=\left(\sum_{i=1}^{m} y_{i}\left(H_{i}^{T} R_{i}^{-1} H_{i}\right)\right)^{-1}=G^{-1}(y)
$$

or all parameters

$$
D(\hat{z}, y)=H G^{-1}(y) H^{T}
$$

where $H_{i}$ is the $i$ th row of $H$.

A PMU is used to acquire a bus voltage phasor $\tilde{V}_{i}=V_{i} \angle \delta_{i}$ and one or more branch current phasors $\tilde{I}_{i j}=I_{i j} \angle \varphi_{i j}$ in a power system. The vector of potential measurements $z=\left(V_{i}, \ldots \delta_{i}, \ldots I_{i j}, \ldots \varphi_{i j}, \ldots\right)^{T}$ is related to the state vector $x=\left(V_{i}, \ldots \delta_{i}, \ldots\right)^{T}$ by a nonlinear function $z(x)$. In order to solve the problem (2)-(4), a linear measurement model similar to (1) needs to be formulated.

\subsection{Polar coordinates formulation}

Let us first consider the original measurement model, when the current and voltage phasors are expressed in polar coordinates. The nonlinear models are generally handled by using their linearized models obtained from the first order Taylor approximation. Applying the linearization technique to $z(x)$ in a neighborhood of a certain state $x^{\prime}$, we have $\Delta z \approx H \Delta x$. In this case, the matrix $H$ is a partial derivative matrix:

$$
H=\frac{\partial z(x)}{\partial x}=\left[\begin{array}{cc}
E & 0 \\
0 & E \\
\frac{\partial I_{i j}}{\partial V} & \frac{\partial I_{i j}}{\partial \delta} \\
\frac{\partial \varphi_{i j}}{\partial V} & \frac{\partial \varphi_{i j}}{\partial \delta}
\end{array}\right]
$$

where $E$ is an identity matrix. The elements of the submatrices corresponding to the current phasors are shown in Table 1. It can be seen than they nonlinearly depend on $x$. Therefore, the solution vector $y^{*}$ of problem (2)-(4), calculated using matrix (7) and a given matrix $R=\operatorname{diag}\left(\sigma_{V_{i}}^{2}, \sigma_{\delta_{i}}^{2}, \sigma_{I_{i j}}^{2}, \sigma_{\varphi_{i j}}^{2}\right)$ for one power system state $x^{\prime}$, may not be optimal for another state $x^{\prime \prime}$.

Table 1. Expressions of partial derivatives for $I_{i j}$ and $\varphi_{i j}$

\begin{tabular}{|c|c|}
\hline$\frac{\partial I_{i j}}{\partial V_{i}}=\frac{1}{3 I_{i j}}\left[V_{i} A-V_{j}\left(C \cos \delta_{i j}+D \sin \delta_{i j}\right)\right]$ & $\frac{\partial \varphi_{i j}}{\partial V_{i}}=-\frac{1}{V_{i} I_{i j}} \frac{\partial I_{i j}}{\partial \delta_{i}}$ \\
\hline$\frac{\partial I_{i j}}{\partial V_{j}}=\frac{1}{3 I_{i j}}\left[V_{j} B-V_{i}\left(C \cos \delta_{i j}+D \sin \delta_{i j}\right)\right]$ & $\frac{\partial \varphi_{i j}}{\partial V_{j}}=\frac{1}{V_{j} I_{i j}} \frac{\partial I_{i j}}{\partial \delta_{i}}$ \\
\hline$\frac{\partial I_{i j}}{\partial \delta_{i}}=\frac{1}{3 I_{i j}}\left[V_{i} V_{j}\left(C \sin \delta_{i j}-D \cos \delta_{i j}\right)\right]$ & $\frac{\partial \varphi_{i j}}{\partial \delta_{i}}=\frac{V_{i}}{I_{i j}} \frac{\partial I_{i j}}{\partial V_{i}}$ \\
\hline$\frac{\partial I_{i j}}{\partial \delta_{j}}=-\frac{\partial I_{i j}}{\partial \delta_{i}}$ & $\frac{\partial \varphi_{i j}}{\partial \delta_{j}}=1-\frac{\partial \varphi_{i j}}{\partial \delta_{i}}$ \\
\hline
\end{tabular}

Note: Table 1 uses the following terms and symbol: $A=\left(g_{i j}+g_{s i j}\right)^{2}+\left(b_{i j}+b_{s i j}\right)^{2}, \quad B=g_{i j}{ }^{2}+b_{i j}{ }^{2}, \quad C=B+$ $g_{i j} g_{s i j}+b_{i j} b_{s i j}, \quad D=b_{i j} g_{s i j}-g_{i j} b_{s i j}, \quad \delta_{i j}=\delta_{i}-\delta_{j}, \quad$ where $y_{i j}=g_{i j}+j b_{i j}$ and $y_{s i j}=g_{s i j}+j b_{s i j}$ denote the series and shunt admittance of branch $i-j$ correspondently.

\subsection{Rectangular coordinates formulation}

The measurement model becomes linear when rectangular coordinates formulation is adopted, so that $\tilde{V}_{i}=V_{R e, i}+j V_{I m, i}, \tilde{I}_{i j}=I_{R e, i j}+j I_{I m, i j}$. In this case, a function which relates the state variables $x=\left(V_{R e, i}, \ldots V_{I m, i}, \ldots\right)^{T}$ to the measurements $z=\left(V_{R e, i}, \ldots V_{I m, i}, \ldots I_{R e, i j}, \ldots I_{I m, i j}, \ldots\right)^{T}$ is linear, $z=H x$, where matrix $H$ contains only unit submatrices and the branch admittances. It would seem that the independence of this matrix from $x$ gives reason to believe that the optimal measurement set, computed using it, is independent of the power system state. However, converting the phasor measurements from polar coordinates into corresponding rectangular coordinates is accompanied by the calculation of the covariance matrix $R$ of real and imaginary parts:

$$
R=\left(\begin{array}{cccc}
\sigma_{V_{\mathrm{Re} i}}^{2} & \sigma_{V_{\mathrm{Re} i} V_{\mathrm{Im}, i}} & 0 & 0 \\
\sigma_{V_{\mathrm{Re} i} V_{\mathrm{Im}, i}} & \sigma_{V_{\mathrm{Im}, i}}^{2} & 0 & 0 \\
0 & 0 & \sigma_{I_{\mathrm{Re} i j}}^{2} & \sigma_{I_{\mathrm{Re} i} I_{\mathrm{Im}, i}} \\
0 & 0 & \sigma_{I_{\mathrm{Re} i} I_{\mathrm{Im}, i}} & \sigma_{I_{\mathrm{Im}, i j}}^{2}
\end{array}\right)
$$

which ensures the identical results of power system state estimation to those when direct measurements of voltages and current magnitudes and phase angles are used. As a rule, the off-diagonal elements of (8) are neglected, which does not lead to a noticeable degradation in the accuracy of the state estimation [14]. Diagonal elements are shown in Table 2. It can be seen that they nonlinearly depend on the power system state; as a result, the optimal solution of problem (2)-(4) depends on the state as well.

Table 2. Expressions of variances in (8)

\begin{tabular}{|l|l|}
\hline$\sigma_{V_{\text {Reti }}}^{2}=\left(\cos \delta_{i}\right)^{2} \sigma_{V_{i}}^{2}+\left(V_{i} \sin \delta_{i}\right)^{2} \sigma_{\delta_{i}}^{2}$ & $\sigma_{I_{\text {Rety }}}^{2}=\left(\cos \varphi_{i j}\right)^{2} \sigma_{I_{i j}}^{2}+\left(I_{i j} \sin \varphi_{i j}\right)^{2} \sigma_{\varphi_{i j}}^{2}$ \\
\hline$\sigma_{V_{\text {thi }}}^{2}=\left(\sin \delta_{i}\right)^{2} \sigma_{V_{i}}^{2}+\left(V_{i} \cos \delta_{i}\right)^{2} \sigma_{\delta_{i}}^{2}$ & $\sigma_{I_{\text {Imaj }}}^{2}=\left(\sin \varphi_{i j}\right)^{2} \sigma_{I_{i j}}^{2}+\left(I_{i j} \cos \varphi_{i j}\right)^{2} \sigma_{\varphi_{i j}}^{2}$ \\
\hline
\end{tabular}


Thus, for any form of phasor measurement representation, the influence of the system nonlinearity on the optimal solution of problem (2)-(4) is traced. A change in steady state parameters leads to a change in the matrix $H(7)$ if polar coordinates formulation is used, and to a change in the matrix $R(8)$ if rectangular coordinates formulation is used. Both of them cause changes in the covariance matrix (5) or (6).

\section{Numerical results and discussions}

The purpose of numerical studies was to reveal how much the power system nonlinearity affects the optimal solution of PMU placement problem. For this, a series of experiments was performed with various system steady state conditions. Next, only the polar coordinates formulation is considered. The rectangular coordinates formulation gives similar results.

\subsection{Experimental conditions}

We consider the problem of optimal placement of 10 multichannel PMUs for state estimation in IEEE 24-bus test system shown in Fig. 1. It is assumed that each multichannel PMU is capable of monitoring all lines incident to the bus where it is located. Therefore, the set of PMU measurements comprises phasors of the bus voltages and branch currents in polar coordinates.

For 24-bus system, a set of operating states was created by changing the system load from 40 to $117 \%$ of the base load specified in the description of the power system, followed by finding the load flow solution which satisfies the system operating limits and minimizes the generation costs. The framework PowerModels.jl [15] was utilized to calculate the optimal power flow. A total of 78 system steady-state conditions were prepared, and for each of them the matrix $H(7)$ was calculated.

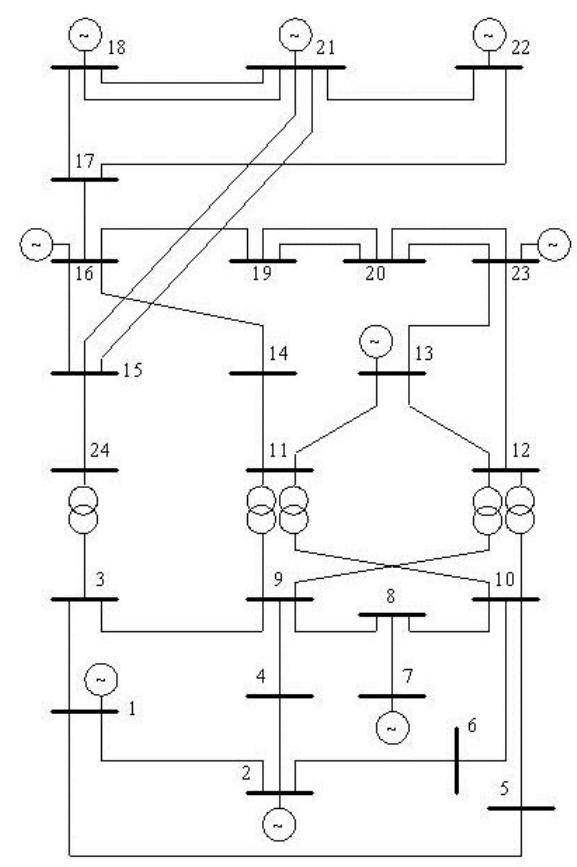

Fig. 1. IEEE 24-bus test system
In order to construct the matrix $R$ the standard deviation of each measurement was calculated considering an error around the value obtained from the power flow base case equal to $0.5 \%$ for magnitudes: $\sigma_{V_{i}}=\frac{1}{3 \cdot 100}\left|V_{i}\right| \cdot 0.5, \sigma_{I_{i j}}=\frac{1}{3 \cdot 100}\left|I_{i j}\right| \cdot 0.5$, and $0.5^{\circ}$ for phase angles: $\sigma_{\delta_{i}}=\sigma_{\varphi_{i j}}=\frac{1}{3 \cdot 100} \cdot 0.5$.

For obtained matrix $R$ and each matrix $H$ corresponding to a different system load, the problem of optimal PMU placement was solved according to the criteria of A-, D-, M-, I- and G-optimality by a method based on mixed integer linear programming [16].

\subsection{PMU placement optimization to maximize the voltage phasor estimation accuracy}

Fig. 2 shows the placement results when using design criteria of A-, D- and M-optimality [10]:

$$
\begin{aligned}
& \Phi_{A}(y)=\frac{1}{n} \operatorname{tr}\left(R_{x}^{-1 / 2} G^{-1}(y) R_{x}^{-1 / 2}\right) \\
& \Phi_{D}(y)=\left(\operatorname{det} G^{-1}(y)\right)^{1 / n} \\
& \Phi_{M}(y)=\max \left(R_{x}^{-1 / 2} G^{-1}(y) R_{x}^{-1 / 2}\right)_{i i}
\end{aligned}
$$

where the matrix $R_{x}$ is formed from the entries of $R$ associated with the state variables $x=\left(V_{i}, \ldots \delta_{i}, \ldots\right)^{T}$. Doptimality criterion corresponds to minimizing the volume of the confidence ellipsoid for the state estimation error. A-optimal PMU placement minimizes the mean squared error in estimating $x$, and M-optimal PMU placement minimizes the worst case variance of the estimation error for $x$.

When using A-optimality criterion, the effect of system operating conditions on the optimal location of the PMUs is not observed. For any system load level, optimization results in the same solution, namely, placing 10 PMUs at buses $1,2,6,8,10,11,16,20,21$, and 24. Note that the estimation error (value of $\Phi_{A}$ at the solution point) depends on the load conditions (Fig. 2a).

In experiments with the D- or M-optimality criterion, 9 different PMUs placements are obtained, which are optimal for different system load levels (Fig. 2b and 2c). These local optimal placements are presented in Table 3 and marked on Fig. 2 with a serial number. In addition to the criterion values that correspond to the optimal placement (solid line), the values corresponding to the worst, minimax, and integral selection of PMU locations are shown. By worst, we mean such a placement configuration out of the nine found options that yields maximum value of the optimality criterion for this load level. From Fig. 2 it can be seen that ill-considered choice of the PMU placement, which is M-optimal for one load level, can lead to a $50 \%$ devaluation of the optimality with another system load level.

Minimax and integral placements are solutions that minimize, respectively, the maximum and average value of the objective function over the whole set of 78 load levels. For D-optimality, such placements are No 3 and No 8 , which are optimal for cases characterized by the 


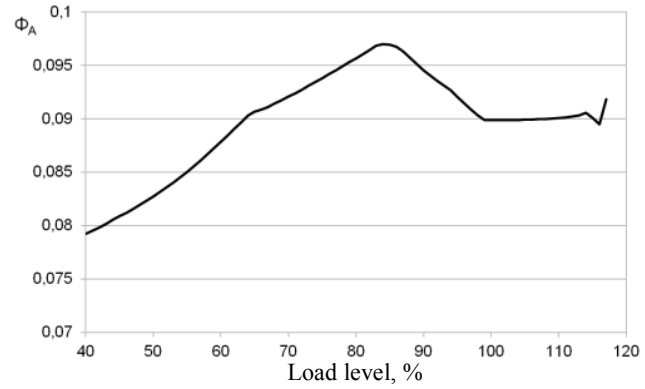

(a)

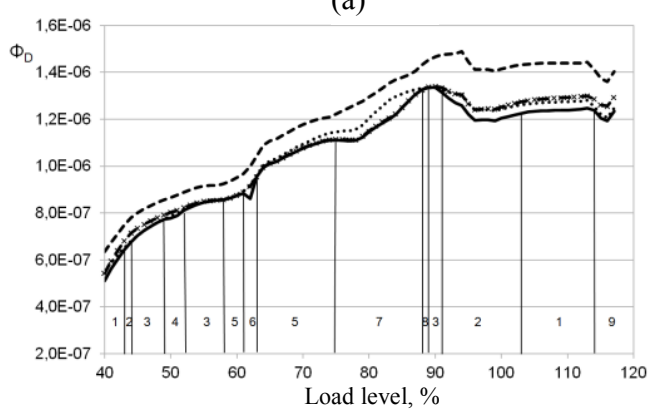

(b)

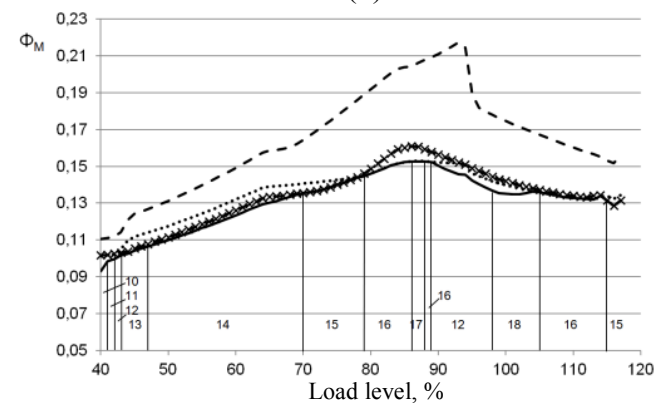

(c)

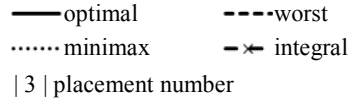

Fig. 2. Results of PMU placement for different levels of system load with using criteria of (a) A-, (b) D-, (c) Moptimality

highest values of the function $\Phi_{D}$ (load is $\left.88-91 \%\right)$. In the case of M-optimality, the minimax No16 and integral No 15 placements are optimal for high load conditions (load is $105 \%$ or more). The latter is consistent with works [11,12], where the selection of measurement locations for minimizing the state estimation error variances in a distribution network is proposed to be made by considering the peak load condition.

\subsection{PMU placement optimization to maximize the voltage and current phasor estimation accuracy}

Fig. 3 shows the results of experiments performed using the I- and G-optimality criteria [10]:

$$
\begin{aligned}
& \Phi_{I}(y)=\frac{1}{m} \operatorname{tr}\left(R^{-1 / 2} H G^{-1}(y) H^{T} R^{-1 / 2}\right) \\
& \Phi_{G}(y)=\max \left(R^{-1 / 2} H G^{-1}(y) H^{T} R^{-1 / 2}\right)_{i i}
\end{aligned}
$$

\begin{tabular}{|c|c|c|c|}
\hline No & PMU buses & No & PMU buses \\
\hline 1 & $\begin{array}{c}1,8,9,10,11,15,16 \\
17,20,21\end{array}$ & 18 & $\begin{array}{c}2,8,9,10,11,16,20 \\
21,22,24\end{array}$ \\
\hline 2 & $\begin{array}{c}1,8,9,10,13,15,16 \\
17,20,21\end{array}$ & 19 & $\begin{array}{c}1,8,9,10,11,15,17 \\
19,21,23\end{array}$ \\
\hline 3 & $\begin{array}{c}2,8,9,10,13,15,16 \\
17,20,21\end{array}$ & 20 & $\begin{array}{c}2,3,5,8,11,12,16 \\
17,20,21\end{array}$ \\
\hline 4 & $\begin{array}{c}2,8,9,10,11,15,17 \\
19,21,23\end{array}$ & 21 & $\begin{array}{c}2,5,7,8,9,13,15 \\
16,20,21\end{array}$ \\
\hline 5 & $\begin{array}{c}2,5,8,9,13,15,16 \\
17,20,21\end{array}$ & 22 & $\begin{array}{c}2,5,8,9,11,15,17 \\
19,21,23\end{array}$ \\
\hline 6 & $\begin{array}{c}2,5,8,9,13,15,16 \\
20,21,23\end{array}$ & 23 & $\begin{array}{c}1,8,9,10,13,16,17 \\
20,21,24\end{array}$ \\
\hline 7 & $\begin{array}{c}2,5,8,9,11,13,15 \\
16,20,21\end{array}$ & 24 & $\begin{array}{c}1,8,9,10,11,15,17 \\
18,19,23\end{array}$ \\
\hline 8 & $\begin{array}{c}1,2,8,9,10,13,15 \\
16,20,21\end{array}$ & 25 & $\begin{array}{c}2,3,5,8,11,12,16 \\
17,18,20\end{array}$ \\
\hline 9 & $\begin{array}{c}2,8,9,10,11,15,16 \\
17,20,21\end{array}$ & 26 & $\begin{array}{c}2,3,8,10,11,12,16 \\
17,20,21\end{array}$ \\
\hline 10 & $\begin{array}{c}1,2,6,8,10,11,16 \\
21,23,24\end{array}$ & 27 & $\begin{array}{c}2,3,8,10,11,12,16 \\
18,20,22\end{array}$ \\
\hline 11 & $\begin{array}{c}1,2,6,8,10,13,16 \\
20,21,24\end{array}$ & 28 & $\begin{array}{c}2,3,5,8,11,12,16 \\
18,20,21\end{array}$ \\
\hline 12 & $\begin{array}{c}1,2,8,10,11,17,19, \\
21,23,24\end{array}$ & 29 & $\begin{array}{c}2,3,5,8,11,13,16 \\
18,20,22\end{array}$ \\
\hline 13 & $\begin{array}{c}1,2,8,10,11,16,21 \\
22,23,24\end{array}$ & 30 & $\begin{array}{c}2,3,7,8,10,13,16 \\
17,20,21\end{array}$ \\
\hline 14 & $\begin{array}{c}1,2,8,9,11,16,20 \\
21,22,24\end{array}$ & 31 & $\begin{array}{c}1,4,7,10,13,15,16, \\
20,21,23\end{array}$ \\
\hline 15 & $\begin{array}{c}1,2,8,9,11,16,21 \\
22,23,24\end{array}$ & 32 & $\begin{array}{c}1,4,8,10,13,15,16, \\
20,21,23\end{array}$ \\
\hline 16 & $\begin{array}{c}1,2,8,9,11,17,19 \\
21,23,24\end{array}$ & 33 & $\begin{array}{c}1,8,9,10,13,16,18 \\
20,22,24\end{array}$ \\
\hline 17 & $\begin{array}{c}2,8,9,10,11,17,19, \\
21,23,24\end{array}$ & & \\
\hline
\end{tabular}

Table 3. Local optimal PMU placements

The PMU placement corresponding to the minimum value of function (12) or (13) provides the best (in the sense of average or worst case error variance) accuracy of estimating both bus voltages and currents over all the branches.

As in the case in D- and M-optimality criteria, different optimal PMU placements are obtained for different load levels. However the type of the dependencies on the load level in Fig.3 differs drastically from those shown in Fig. 2. In some operating conditions (for example, in the range of $57-63 \%$ load), the estimation error (in the sense of criterion I- or Goptimality) may manifold increase even with the optimal selection of PMU locations. Moreover, the devaluation of optimality in the case of ill-considered choice of the steady state used to solve the problem of the PMU placement reaches 10 - and 100-fold increase in the objective function values, respectively, with using the criterion of I- and G-optimality. Detailed analysis of these cases shows that the source of the peaks is lightly loaded branches.

When current $I_{i j}$ of branch $i-j$ is close to zero and $V_{i} \approx V_{j}$ and $\delta_{i} \approx \delta_{j}$, then the partial derivatives of phase angle $\varphi_{i j}$ with respect to state variables (bus voltage magnitudes and angles) can tend to infinity [14]. If the current phasor $\tilde{I}_{i j}=I_{i j} \angle \varphi_{i j}$ is not measured, the angle 


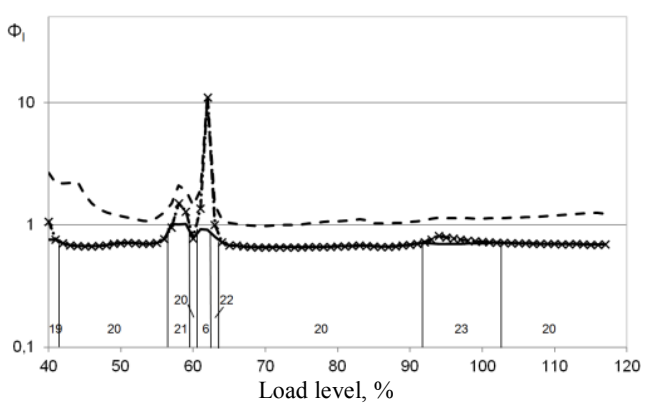

a)

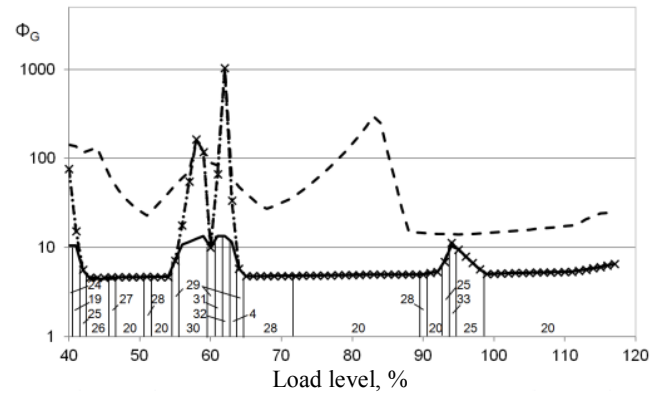

b)

Fig. 3. Results of PMUs placement for different levels of system load with using criteria (a) I- and (b) G-optimality (for designations see Fig.2)

estimation error is not limited. As an illustration, Fig. 4 shows equal confidence ellipsoids for estimates of two current phasors. For current $\tilde{I}_{l}$, the angle estimation error is in the range $\pm \Delta \varphi_{1}$. For current $\tilde{I}_{2}$, the magnitude of which is close to zero, the angle estimate can take a value from -180 to $180^{\circ}$.

The described situation is most noticeable at $61-63 \%$ load. In these operating states, currents of lines 20-23 are near zero which leads to increase in estimation errors of angles $\varphi_{23-20}$. They can only be restricted by placing a PMU at bus 23 and it happens according to the results of solving the optimal PMU placement problem. Placement designs that are optimal for other system load conditions do not provide for the installation of a PMU at bus 23, and their use for state estimation at $61-63 \%$ load gives growth in normalized variance of the estimates of angles $\varphi_{23-20}$ equal up to 1032.7 . Similar situations occur at $40-$ $41 \%$ load (currents of lines $1-3$ and 3-9 are near zero) and at $57-60 \%$ load (current of line $7-8$ is near zero).

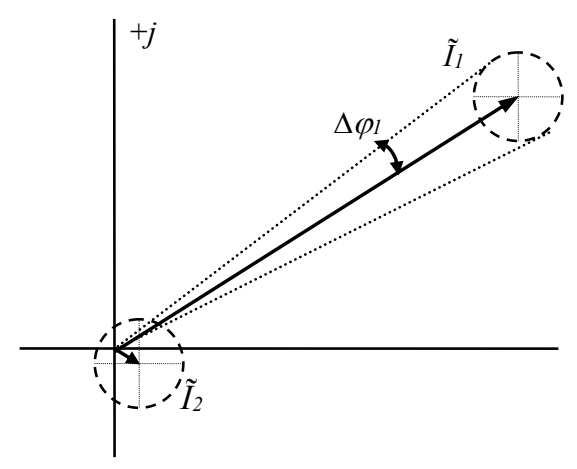

Fig. 4. Illustration of an angular error in current phasor
It should be noted that the value of phase angle error of zero current phasor is not relevant. To avoid the influence of zero current phasors on the choice of PMU locations, it is necessary to preclude using steady states with lightly loaded branches when solving the optimal PMU placement problem. Following this proposal, Fig. 3 shows the values of $\Phi_{I}$ and $\Phi_{G}$ corresponding to the minimax and integral placements, which were obtained by excluding the states with the load levels of 40-41 and $57-63 \%$. The corresponding placement No 20 is optimal for almost all other levels of system load, both for criteria of I- and G-optimality.

\section{Conclusion}

The solution to the problem of optimal PMU placement, aimed at ensuring the maximum accuracy of the power system state estimation, depends on the system steady state used for optimization. Disregard of nonlinearity effects when choosing locations of the PMUs according to the criteria of D-, M-, I- or G-optimality can lead to a noticeable loss of accuracy (relative to what is expected) in some operating states of the power system. This raises the problem of finding the PMU placement design that optimizes the estimation accuracy among all possible system state. Preliminary results have shown that minimax or integral selection the PMU placement locations can be a promising solution. Therein, in the case of using the I- or G-optimality criteria, the operating states with lightly loaded branches should be excluded. The influence of the system nonlinearity on A-optimal solutions has not been revealed, which allows using the existing optimization models based on considering a single steady state of power system to find the A-optimal PMU placement.

\section{References}

1. A.G. Phadke, J. S. Thorp, Synchronized phasor measurement and their applications (Springer, NY, 2008)

2. I.N. Kolosok, E.S. Korkina, E.A. Buchinskii, Elektrichestvo, 3, 4 (2014) [in Russian]

3. L. Zanni, A. Derviškadić, M. Pignati, C. Xu, et al., Electric Power System Research, 21st PSCC, 189, (2020)

4. M.V. Khokhlov, N.E. Gotman, Methodological Problems in Reliability Study of Large Energy Systems, 68, 495 (2017) [in Russian]

5. Transient mode monitoring system of the Unified Energy System of Russia (2020) [Online] Available: https://www.smpr.technology

6. V.V. Fedorov, Theory of optimal experiments (Academic Press, NY, 1971)

7. V. Kekatos, G.B. Giannakis, B. Wollenberg, IEEE Trans. on Pow. Syst. 27 (3), 1521 (2012)

8. P. Yang, Z. Tan, A. Wiesel, A. Nehorai, IEEE Trans. on Pow. Deliv. 30 (2), 914 (2015) 
9. I.I. Golub, M.V. Khokhlov, Elektrichestvo, 1, 26 (2015) [in Russian]

10. M.V. Khokhlov, Methodological Problems in Reliability Study of Large Energy Systems, 2, 382 (2018) DOI: 10.13140/RG.2.2.12656.07685 [in Russian]

11. M. G. Damavandi, V. Krishnamurthy, J. R. Martí, IEEE Trans. on Smart Grid, 6 (4), 1972 (2015)

12. T.C. Xygkis, G.N. Korres, N.M. Manousakis, IEEE Trans. on Smart Grid, 9 (2), 1452 (2018)

13. T. C. Xygkis, G. N. Korres, N. M. Manousakis, Proc. IEEE Milan PowerTech, 1 (2019)

14. M.V. Khokhlov, 3rd Int. Scient. and Tech. Conf., 1, 795 (2012) DOI: 10.13140/RG.2.1.4591.6642 [in Russian]

15. C. Coffrin, R. Bent, K. Sundar, Y. Ng, M. Lubin, 20th PSCC, 1 (2018)

16. M.V. Khokhlov, Actual Problems, Directions and Mechanisms of Development of the Productive Forces of the North - 2020, 2, 152 (2020) [in Russian] 